\title{
ALGEBRAIC CYCLES AND INTERSECTIONS OF A QUADRIC AND A CUBIC
}

\author{
ROBERT LATERVEER
}

\begin{abstract}
Let $Y$ be a smooth complete intersection of a quadric and a cubic in $\mathbb{P}^{n}$, with $n$ even. We show that $Y$ has a multiplicative Chow-Künneth decomposition, in the sense of Shen-Vial. As a consequence, the Chow ring of (powers of) $Y$ displays K3-like behaviour. As a by-product of the argument, we also establish a multiplicative Chow-Künneth decomposition for the resolution of singularities of a general nodal cubic hypersurface of even dimension.
\end{abstract}

\section{INTRODUCTION}

Given a smooth projective variety $Y$ over $\mathbb{C}$, let $A^{i}(Y):=C H^{i}(Y)_{\mathbb{Q}}$ denote the Chow groups of $Y$ (i.e. the groups of codimension $i$ algebraic cycles on $Y$ with $\mathbb{Q}$-coefficients, modulo rational equivalence [12]). The intersection product defines a ring structure on $A^{*}(Y)=\bigoplus_{i} A^{i}(Y)$, the Chow ring of $Y$. In the case of $\mathrm{K} 3$ surfaces, this ring structure has a remarkable property:

Theorem 1.1 (Beauville-Voisin [3]). Let $S$ be a $K 3$ surface. The $\mathbb{Q}$-subalgebra

$$
R^{*}(S):=\left\langle A^{1}(S), c_{j}(S)\right\rangle \quad \subset A^{*}(S)
$$

injects into cohomology under the cycle class map.

Motivated by the particular behaviour of Chow rings of K3 surfaces and of abelian varieties [1], Beauville [2] has conjectured that for certain special varieties, the Chow ring should admit a multiplicative splitting. To make concrete sense of Beauville's elusive "splitting property" conjecture, Shen-Vial [32] introduced the concept of multiplicative Chow-Künneth decomposition. It is something of a challenge to understand the class of special varieties admitting such a decomposition: abelian varieties, K3 surfaces, cubic hypersurfaces, hyperelliptic curves and some Fano varieties are in this class (but not all Fano varieties), cf. subsection 2.2 below.

The main result of the present paper aims to contribute to this program:

Theorem (=Theorem 4.1). Let $Y \subset \mathbb{P}^{n}(\mathbb{C})$ be a smooth complete intersection of a quadric and a cubic, where $n$ is even. Then $Y$ has a (generically defined) multiplicative Chow-Künneth decomposition.

The assumption on $n$ is there for good reason: indeed, the statement of the theorem is false for $n=3$ (cf. Remark 3.9). The question what happens for $n>3$ odd is an interesting open problem.

Key words and phrases. Algebraic cycles, Chow group, motive, Bloch-Beilinson filtration, Beauville's "splitting property" conjecture, multiplicative Chow-Künneth decomposition, Fano varieties, tautological ring.

2020 Mathematics Subject Classification: 14C15, 14C25, 14C30.

Supported by ANR grant ANR-20-CE40-0023. 
In proving Theorem 4.1, we rely on the connection between a general $Y$ and a nodal cubic $n$-fold, and we apply instances of the Franchetta property (cf. Section 3 below).

Theorem 4.1 implies that the Chow ring of $Y$ behaves just like that of $\mathrm{K} 3$ surfaces, i.e. the image of the intersection product is as small as possible:

Corollary (=Corollary 5.1). Let $Y \subset \mathbb{P}^{n}$ be as in Theorem 4.1] Then

$$
\operatorname{Im}\left(A^{i}(Y) \otimes A^{j}(Y) \rightarrow A^{i+j}(Y)\right)=\mathbb{Q}\left[h^{i+j}\right] \quad \forall i, j>0
$$

(where $h \in A^{1}(Y)$ denotes the hyperplane class).

Using Theorem 4.1, we can also prove a result concerning the tautological ring, which is a certain subring of the Chow ring of powers of $Y$ :

Corollary (=Corollary 5.4). Let $Y \subset \mathbb{P}^{n}$ be as in Theorem 4.1] and $m \in \mathbb{N}$. Let

$$
R^{*}\left(Y^{m}\right):=\left\langle\left(p_{i}\right)^{*}(h),\left(p_{i j}\right)^{*}\left(\Delta_{Y}\right)\right\rangle \subset A^{*}\left(Y^{m}\right)
$$

be the $\mathbb{Q}$-subalgebra generated by pullbacks of the polarization $h \in A^{1}(Y)$ and pullbacks of the diagonal $\Delta_{Y} \in A^{n-2}(Y \times Y)$. The cycle class map induces injections

$$
R^{*}\left(Y^{m}\right) \hookrightarrow H^{*}\left(Y^{m}, \mathbb{Q}\right) \quad \forall m \leq 2 \operatorname{dim} H^{n-2}(Y, \mathbb{Q})-1
$$

Again, this is similar to existing results for hyperelliptic curves and for K3 surfaces (cf. [34], [35], [38] and Remark 5.5] below).

Theorem 4.1 also has consequences for the Chow ring of resolutions of nodal cubic hypersurfaces:

Corollary (=Corollary 5.6). Let $\bar{Z} \subset \mathbb{P}^{n+1}(\mathbb{C})$ be a general nodal cubic hypersurface, where $n$ is even. Then there is a resolution of singularities $Z \rightarrow \bar{Z}$ such that $Z$ has a multiplicative Chow-Künneth decomposition.

Conventions. In this article, the word variety will refer to a reduced irreducible scheme of finite type over $\mathbb{C}$. A subvariety is a (possibly reducible) reduced subscheme which is equidimensional.

All Chow groups are with rational coefficients: we will denote by $A_{j}(Y)$ the Chow group of $j$-dimensional cycles on $Y$ with $\mathbb{Q}$-coefficients; for $Y$ smooth of dimension $n$ the notations $A_{j}(Y)$ and $A^{n-j}(Y)$ are used interchangeably. The notations $A_{h o m}^{j}(Y)$ and $A_{A J}^{j}(Y)$ will be used to indicate the subgroup of homologically trivial (resp. Abel-Jacobi trivial) cycles. For a morphism $f: X \rightarrow Y$, we will write $\Gamma_{f} \in A_{*}(X \times Y)$ for the graph of $f$.

The contravariant category of Chow motives (i.e., pure motives with respect to rational equivalence as in [31], [28]) will be denoted $\mathcal{M}_{\text {rat }}$.

\section{PRELiminaries}

\subsection{Intersections of a quadric and a cubic.}


Proposition 2.1. Let $\bar{Z}$ be the nodal cubic $n$-fold defined as

$$
\bar{Z}:=\left\{y \in \mathbb{P}^{n+1} \mid f\left(x_{0}, \ldots, x_{n}\right)+q\left(x_{0}, \ldots, x_{n}\right) \cdot x_{n+1}=0\right\} \quad \subset \mathbb{P}^{n+1},
$$

where $f$ and $g$ are general polynomials of degree 3 resp. 2. Let $\phi: Z \rightarrow \bar{Z}$ be the blow-up with center the node $P=[0: 0: \ldots: 0: 1]$. Then $Z$ is isomorphic to the blow-up $\psi$ of $\mathbb{P}^{n}$ with center

$$
Y:=\left\{y \in \mathbb{P}^{n} \mid f(y)=q(y)=0\right\} \quad \subset \mathbb{P}^{n} .
$$

Proof. This is probably well-known. The case $n=7$ is contained in [15, Section 5.7]; the general case is similar. The point is that projection from the node $P$ defines a birational map $p$ from $\bar{Z}$ to $\left\{x_{n+1}=0\right\} \cong \mathbb{P}^{n}$. The blow-up $\phi$ provides a resolution of indeterminacy of this birational map; there is a commutative diagram

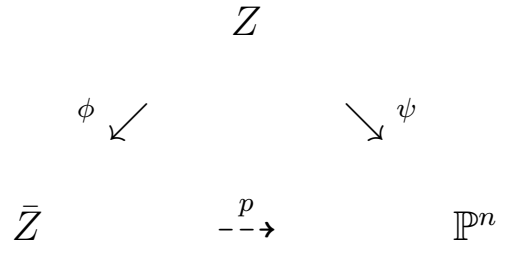

Remark 2.2. Let notation be as in Proposition 2.1. In case $n=4, Y$ is a $\mathrm{K} 3$ surface associated to a nodal cubic fourfold; this case is related to singular hyperkähler varieties as shown in [26], cf. notably [26, Lemma 3.3] and also [14, Chapter 6 section 1.2].

In case $n=7, Y$ is a fivefold of "CY3-type" (i.e. the Hodge diamond of $Y$ looks like that of a Calabi-Yau threefold, cf. [15], [16]), and Proposition 2.1 relates $Y$ to a nodal cubic sevenfold (which is another variety of CY3-type).

\subsection{MCK decomposition.}

Definition 2.3 (Murre [27]). Let $X$ be a smooth projective variety of dimension $n$. We say that $X$ has a $C K$ decomposition if there exists a decomposition of the diagonal

$$
\Delta_{X}=\pi_{X}^{0}+\pi_{X}^{1}+\cdots+\pi_{X}^{2 n} \text { in } A^{n}(X \times X),
$$

such that the $\pi_{X}^{i}$ are mutually orthogonal idempotents and $\left(\pi_{X}^{i}\right)_{*} H^{*}(X, \mathbb{Q})=H^{i}(X, \mathbb{Q})$.

(NB: "CK decomposition" is shorthand for "Chow-Künneth decomposition".)

Remark 2.4. The existence of a CK decomposition for any smooth projective variety is part of Murre's conjectures [27], [17].

Definition 2.5 (Shen-Vial [32]). Let $X$ be a smooth projective variety of dimension $n$. Let $\Delta_{X}^{s m} \in A^{2 n}(X \times X \times X)$ be the class of the small diagonal

$$
\Delta_{X}^{s m}:=\{(x, x, x) \mid x \in X\} \subset X \times X \times X .
$$

An MCK decomposition is a CK decomposition $\left\{\pi_{X}^{i}\right\}$ of $X$ that is multiplicative, i.e. it satisfies

$$
\pi_{X}^{k} \circ \Delta_{X}^{s m} \circ\left(\pi_{X}^{i} \times \pi_{X}^{j}\right)=0 \text { in } A^{2 n}(X \times X \times X) \text { for all } i+j \neq k .
$$

(NB: "MCK decomposition" is shorthand for "multiplicative Chow-Künneth decomposition".) 
Remark 2.6. The small diagonal (seen as a correspondence from $X \times X$ to $X$ ) induces the multiplication morphism

$$
\Delta_{X}^{s m}: h(X) \otimes h(X) \rightarrow h(X) \text { in } \mathcal{M}_{\text {rat }} .
$$

Suppose $X$ has a CK decomposition

$$
h(X)=\bigoplus_{i=0}^{2 n} h^{i}(X) \text { in } \mathcal{M}_{\text {rat }} .
$$

By definition, this decomposition is multiplicative if for any $i, j$ the composition

$$
h^{i}(X) \otimes h^{j}(X) \rightarrow h(X) \otimes h(X) \stackrel{\Delta_{X}^{s m}}{\longrightarrow} h(X) \text { in } \mathcal{M}_{\text {rat }}
$$

factors through $h^{i+j}(X)$.

If $X$ has an MCK decomposition, then setting

$$
A_{(j)}^{i}(X):=\left(\pi_{X}^{2 i-j}\right)_{*} A^{i}(X),
$$

one obtains a bigraded ring structure on the Chow ring: that is, the intersection product sends $A_{(j)}^{i}(X) \otimes A_{\left(j^{\prime}\right)}^{i^{\prime}}(X)$ to $A_{\left(j+j^{\prime}\right)}^{i+i^{\prime}}(X)$.

It is expected that for any $X$ with an MCK decomposition, one has

$$
A_{(j)}^{i}(X) \stackrel{? ?}{=} 0 \text { for } j<0, \quad A_{(0)}^{i}(X) \cap A_{h o m}^{i}(X) \stackrel{? ?}{=} 0 ;
$$

this is related to Murre's conjectures $\mathrm{B}$ and $\mathrm{D}$, that have been formulated for any CK decomposition [27].

The property of having an MCK decomposition is severely restrictive, and is closely related to Beauville's "splitting property conjecture" [2]. To give an idea: hyperelliptic curves have an MCK decomposition [32, Example 8.16], but the very general curve of genus $\geq 3$ does not have an MCK decomposition [9, Example 2.3]. As for surfaces: a smooth quartic in $\mathbb{P}^{3}$ has an MCK decomposition, but a very general surface of degree $\geq 7$ in $\mathbb{P}^{3}$ should not have an MCK decomposition [9, Proposition 3.4]. For more detailed discussion, and examples of varieties with an MCK decomposition, we refer to [32, Section 8], as well as [36], [33], [10], [19], [25], [20], [21], [22], [9], [23], [24], [29].

Proposition 2.7 (Shen-Vial [33]). Let $M$ be a smooth projective variety, and let $f: \widetilde{M} \rightarrow M$ be the blow-up with center a smooth closed subvariety $N \subset M$. Assume that

(1) $M$ and $N$ have an MCK decomposition;

(2) the Chern classes of the normal bundle $\mathcal{N}_{N / M}$ are in $A_{(0)}^{*}(N)$;

(3) the graph of the inclusion morphism $N \rightarrow M$ is in $A_{(0)}^{*}(N \times M)$;

(4) the Chern classes $c_{j}\left(T_{M}\right)$ are in $A_{(0)}^{*}(M)$.

Then $\widetilde{M}$ has an MCK decomposition, the Chern classes $c_{j}\left(T_{\widetilde{M}}\right)$ are in $A_{(0)}^{*}(\widetilde{M})$, and the graph $\Gamma_{f}$ is in $A_{(0)}^{*}(\widetilde{M} \times M)$.

Proof. This is [33, Proposition 2.4]. (NB: in loc. cit., $M$ and $N$ are required to have a selfdual MCK decomposition; however, the self-duality is actually a redundant hypothesis, cf. [11, Section 6].) 


\section{THE FRANCHETTA PROPERTY}

\subsection{Definition.}

Definition 3.1. Let $\mathcal{X} \rightarrow B$ be a smooth projective morphism, where $\mathcal{X}, B$ are smooth quasiprojective varieties. We say that $\mathcal{X} \rightarrow B$ has the Franchetta property in codimension $j$ if the following holds: for every $\Gamma \in A^{j}(\mathcal{X})$ such that the restriction $\left.\Gamma\right|_{X_{b}}$ is homologically trivial for the very general $b \in B$, the restriction $\left.\Gamma\right|_{b}$ is zero in $A^{j}\left(X_{b}\right)$ for all $b \in B$.

We say that $\mathcal{X} \rightarrow B$ has the Franchetta property if $\mathcal{X} \rightarrow B$ has the Franchetta property in codimension $j$ for all $j$.

This property is studied in [30], [4], [7], [8].

Definition 3.2. Given a family $\mathcal{X} \rightarrow B$ as above, with $X:=X_{b}$ a fiber, we write

$$
G D A_{B}^{j}(X):=\operatorname{Im}\left(A^{j}(\mathcal{X}) \rightarrow A^{j}(X)\right)
$$

for the subgroup of generically defined cycles. (In a context where it is clear to which family we are referring, the index $B$ will sometimes be suppressed from the notation.)

With this notation, the Franchetta property amounts to saying that $G D A^{*}(X)$ injects into cohomology, under the cycle class map.

\subsection{The families.}

Notation 3.3. Let $\mathcal{Y} \rightarrow B$ denote the universal family of smooth $n$-2-dimensional complete intersections of a quadric and a cubic over $B \subset \bar{B}:=\mathbb{P} H^{0}\left(\mathbb{P}^{n}, \mathcal{O}_{\mathbb{P}^{n}}(3) \oplus \mathcal{O}_{\mathbb{P}^{n}}(2)\right)$, the corresponding parameter space.

Notation 3.4. Let $B$ be as in Notation 3.3 , and let $\overline{\mathcal{Z}} \subset \mathbb{P}^{n+1} \times B$ denote the universal family of nodal cubic $n$-folds defined by an equation

$$
f\left(x_{0}, \ldots, x_{n}\right)+q\left(x_{0}, \ldots, x_{n}\right) \cdot x_{n+1}=0
$$

as in Proposition 2.1.

Let $\phi: \widetilde{\mathbb{P}}^{n+1} \rightarrow \mathbb{P}^{n+1}$ be the blow-up with center $P=[0: 0: \ldots: 0: 1]$, and let $\mathcal{Z} \subset \widetilde{\mathbb{P}}^{n+1} \times B$ denote the blow-up of $\overline{\mathcal{Z}}$ with center $p_{1}^{-1}(P)$. Let $B_{0} \subset B$ be the (non-empty) Zariski open such that the fibers of $\mathcal{Z} \rightarrow B_{0}$ are smooth.

Lemma 3.5. The varieties $\mathcal{Y}$ and $\mathcal{Z}$ are smooth.

Proof. Let $\overline{\mathcal{Y}} \supset \mathcal{Y}$ denote the Zariski closure of $\mathcal{Y}$ in $\mathbb{P}^{n} \times \bar{B}$. As $\mathcal{O}_{\mathbb{P}^{n}}(3) \oplus \mathcal{O}_{\mathbb{P}^{n}}(2)$ is base-point free, the projection $\mathcal{Y} \rightarrow \mathbb{P}^{n}$ has the structure of a projective bundle, hence is smooth.

As for $\mathcal{Z}$, according to Proposition 2.1 the Zariski closure of $\mathcal{Z}$ is isomorphic to the blow-up of $\mathbb{P}^{n} \times \bar{B}$ with center $\overline{\mathcal{Y}}$, hence is smooth.

\subsection{Franchetta for $Y$.}

Proposition 3.6. Let $\mathcal{Y} \rightarrow B$ be the universal family of smooth complete intersections of bidegree $(2,3)$ in $\mathbb{P}^{n}$ (Notation 3.3). The family $\mathcal{Y} \rightarrow B$ has the Franchetta property, for any $n$. 
Proof. This is easy. We have already seen (proof of Lemma 3.5) that $\overline{\mathcal{Y}} \rightarrow \mathbb{P}^{n}$ is a projective bundle. Using the projective bundle formula, and reasoning as in [30] and [7], this implies that

$$
G D A_{B}^{*}(Y)=\operatorname{Im}\left(A^{*}\left(\mathbb{P}^{n}\right) \rightarrow A^{*}(Y)\right)=\langle h\rangle,
$$

where $h \in A^{1}(Y)$ is the restriction of a hyperplane section. It follows that $G D A_{B}^{*}(Y)$ injects into cohomology.

\subsection{Franchetta for $Y^{2}$.}

Proposition 3.7. Let $\mathcal{Y} \rightarrow B$ be as above, and assume $n$ even. Then the family $\mathcal{Y}^{2 / B} \rightarrow B$ has the Franchetta property.

Proof. We note that $\mathcal{O}_{\mathbb{P}^{n}}(2)$ and $\mathcal{O}_{\mathbb{P}^{n}}(3)$ are (not only base-point free but even) very ample, which means that the set-up verifies condition $\left(*_{2}\right)$ of [7]. Then applying the stratified projective bundle argument as in loc. cit. (in the precise form of [8, Proposition 2.6]), we find that

$$
G D A^{*}\left(Y^{2}\right)=\left\langle h, \Delta_{Y}\right\rangle \text {. }
$$

Let us now check that the right-hand side injects into cohomology. Using Lemma 3.8 below, $G D A^{j}\left(Y^{2}\right)$ for $j \neq n-2$ is decomposable, i.e.

$$
G D A^{j}\left(Y^{2}\right) \subset G D A^{*}(Y) \otimes G D A^{*}(Y) \forall j \neq n-2 .
$$

Thus for $j \neq n-2$, the injectivity reduces to Proposition 3.6 ,

For $j=n-2$, one observes that the class of the diagonal in $H^{2 n-4}(Y \times Y, \mathbb{Q})$ can not be expressed as a decomposable cycle (for otherwise $Y$ would not have any transcendental cohomology, which is absurd), and so we reduce again to Proposition 3.6.

Lemma 3.8. Let $Y \subset \mathbb{P}^{n}$ be a smooth complete intersection of a cubic and a quadric, where $n$ is even. Then

$$
\Delta_{Y} \cdot\left(p_{i}\right)^{*}(h)=\sum \frac{1}{6}\left(p_{1}\right)^{*}\left(h^{k}\right) \cdot\left(p_{2}\right)^{*}\left(h^{n-1-k}\right) \text { in } A^{n-1}(Y \times Y) .
$$

To prove the lemma, we write $Y=Y^{\prime} \cap Q$, where $Y^{\prime}$ and $Q$ are a cubic resp. a quadric. Up to shrinking the base $B$, we may assume $Y^{\prime}$ and $Q$ are smooth. Let $\iota: Y \hookrightarrow Q$ denote the inclusion morphism. Then $\Delta_{Y} \cdot\left(p_{i}\right)^{*}(h)$ is equal to

$$
\frac{1}{3}{ }^{t} \Gamma_{\iota} \circ \Gamma_{\iota}=\frac{1}{3}(\iota \times \iota)^{*}\left(\Delta_{Q}\right) \text { in } A^{n-1}(Y \times Y) .
$$

But $Q$, being an odd-dimensional smooth quadric, has $A^{j}(Q)=\mathbb{Q}$ for all $j$ and so $\Delta_{Q}$ can be written as

$$
\Delta_{Q}=\sum \frac{1}{2}\left(p_{1}\right)^{*}\left(h^{k}\right) \cdot\left(p_{2}\right)^{*}\left(h^{n-1-k}\right) \text { in } A^{n-1}(Q \times Q) .
$$

The lemma, and the proposition, are proven.

Remark 3.9. The equality of Lemma 3.8 is remarkable, because this equality may fail for $n$ odd. Indeed, in case $Y \subset \mathbb{P}^{m}$ is a smooth hypersurface (of any degree), there is equality

$$
\Delta_{Y} \cdot\left(p_{i}\right)^{*}(h)=\sum a_{k}\left(p_{1}\right)^{*}\left(h^{k}\right) \cdot\left(p_{2}\right)^{*}\left(h^{m-k}\right) \text { in } A^{m}(Y \times Y),
$$


with $a_{k} \in \mathbb{Q}$, as follows from the excess intersection formula. On the other hand, in case $Y \subset \mathbb{P}^{m}$ is a complete intersection of codimension at least 2, in general there is no equality of the form (11). Indeed, let $C$ be a very general curve of genus $g \geq 4$. The Faber-Pandharipande cycle

$$
F P(C):=\Delta_{C} \cdot\left(p_{j}\right)^{*}\left(K_{C}\right)-\frac{1}{2 g-2} K_{C} \times K_{C} \in A^{2}(C \times C) \quad(j=1,2)
$$

is homologically trivial but non-zero in $A^{2}(C \times C)$ [13], [37] (this cycle $F P(C)$ is the "interesting 0 -cycle" in the title of [13]). In particular, for the very general complete intersection $Y \subset \mathbb{P}^{3}$ of bidegree $(2,3)$, the cycle

$$
F P(Y):=\Delta_{Y} \cdot\left(p_{j}\right)^{*}(h)-\frac{1}{6} h \times h \quad \in A^{2}(Y \times Y)
$$

is homologically trivial but non-zero, and so there cannot exist an equality of the form (1) for $Y$.

This is intimately related to MCK decompositions. Indeed, if the curve $Y$ has an MCK decomposition which is generically defined, then $F P(Y) \in A_{(0)}^{2}(Y \times Y) \cong \mathbb{Q}$ and so $F P(Y)$ would be zero.

Corollary 3.10. Let $\mathcal{Z} \rightarrow B_{0}$ be as in Notation 3.4 and assume $n$ even. Then the family $\mathcal{Z}^{2 / B_{0}} \rightarrow B_{0}$ has the Franchetta property.

Proof. The relation between $Z$ and $Y$ of Proposition 2.1 is obviously generically defined (with respect to $B_{0}$ ), and so there is a generically defined isomorphism of motives

$$
h(Z) \cong h(Y)(-1) \oplus \bigoplus_{k=0}^{n} \mathbb{1}(-k) \text { in } \mathcal{M}_{\text {rat }} .
$$

This induces a commutative diagram

$$
\begin{array}{cc}
G D A_{B_{0}}^{j}\left(Z^{2}\right) \cong G D A_{B_{0}}^{j-2}\left(Y^{2}\right) \oplus \bigoplus G D A_{B_{0}}^{*}(Y) \oplus \mathbb{Q}^{r} \\
\downarrow & \downarrow \\
\downarrow & \\
H^{2 j}\left(Z^{2}, \mathbb{Q}\right) \cong & \cong H^{2 j-4}\left(Y^{2}, \mathbb{Q}\right) \oplus \bigoplus H^{*}(Y, \mathbb{Q}) \oplus \mathbb{Q}^{r}
\end{array}
$$

The corollary now follows from Propositions 3.6 and 3.7 .

\subsection{Franchetta for $Y^{3}$ in codimension $\leq 2 n-4$.}

Proposition 3.11. Let $\mathcal{Y} \rightarrow B$ be as above, and assume $n$ even. Then the family $\mathcal{Y}^{3 / B} \rightarrow B$ has the Franchetta property in codimension $\leq 2 n-4$.

Proof. We exploit the relation between $Y$ and $Z$ of Proposition 2.1, The isomorphism of motives (2) induces a commutative diagram

$$
\begin{array}{ccc}
G D A_{B_{0}}^{j}\left(Y^{3}\right) & \hookrightarrow & G D A_{B_{0}}^{j+3}\left(Z^{3}\right) \\
\downarrow & & \downarrow \\
H^{2 j}\left(Y^{3}, \mathbb{Q}\right) & \hookrightarrow & H^{2 j+6}\left(Z^{3}, \mathbb{Q}\right)
\end{array}
$$


This diagram implies that Proposition 3.11 is a consequence of the following result:

Proposition 3.12. Let $\mathcal{Z} \rightarrow B_{0}$ be as in Notation 3.4 and assume $n$ even. The family $\mathcal{Z}^{3 / B_{0}} \rightarrow$ $B_{0}$ has the Franchetta property in codimension $\leq 2 n-1$.

It remains to prove Proposition 3.12, Let $\phi: \widetilde{\mathbb{P}}^{n+1} \rightarrow \mathbb{P}^{n+1}$ denote the blow-up with center $P$ and exceptional divisor $E$. The family $\mathcal{Z} \rightarrow B_{0}$ can be interpreted as the universal family of hypersurfaces associated to the (very ample) line bundle

$$
L:=\phi^{*} \mathcal{O}_{\mathbb{P}^{n+1}}(3) \otimes \mathcal{O}_{\widetilde{\mathbb{P}}^{n+1}}(-2 E)
$$

on $\widetilde{\mathbb{P}}^{n+1}$.

Let us now convince ourselves that $\left(\widetilde{\mathbb{P}}^{n+1}, L\right)$ has property $\left(*_{3}\right)$ of [7] (which means that 3 distinct points of $\widetilde{\mathbb{P}}^{n+1}$ impose 3 independent conditions on the parameter space $B_{0}$ ). Three distinct points outside of $E$ impose independent conditions on sections of $L$, because 3 distinct points impose independent conditions on cubics in $\mathbb{P}^{n+1}$. Three distinct points contained in $E$ impose independent conditions on sections of $L$, because 3 distinct points impose independent conditions on quadrics in $\mathbb{P}^{n}$ (note that the intersection of $Z$ with the exceptional divisor $E \cong \mathbb{P}^{n}$ is the quadric defined by $q=0$, where $q$ is as in Proposition 2.1). Taking a combination of points in $E$ and points outside $E$, things get even easier: the points in $E$ only impose conditions on the quadric part $q$ of the equation of $\bar{Z}$ in Proposition 2.1, whereas points outside of $E$ only impose conditions on the cubic part $f$ of the equation of $\bar{Z}$. This shows that $\left(\widetilde{\mathbb{P}}^{n+1}, L\right)$ has property $\left(*_{3}\right)$.

Applying the stratified projective bundle argument of [7] (cf. also [8, Proposition 2.6] for the precise form used here), we find that

$$
G D A_{B_{0}}^{*}\left(Z^{3}\right)=\left\langle\left(p_{i}\right)^{*}(h),\left(p_{j}\right)^{*}(E),\left(p_{k l}\right)^{*}\left(\Delta_{Z}\right)\right\rangle
$$

where $h, E \in A^{1}(Z)$ denote the restriction of $\phi^{*}(h)$ resp. the exceptional divisor $E$ to $Z$.

We note that when $j \leq 2 n-1$, an element in $G D A^{j}\left(Z^{3}\right)$ cannot involve a product of 2 diagonals, and so

$$
G D A^{j}\left(Z^{3}\right) \subset \sum G D A^{*}\left(Z^{2}\right) \otimes G D A^{*}(Z) \quad \forall j \leq 2 n-1 .
$$

As these summands go to different pieces of the Künneth decomposition in cohomology, Proposition 3.12 now follows from Proposition 3.10 .

\section{MAIN RESUlT}

Theorem 4.1. Let $Y \subset \mathbb{P}^{n}$ be a smooth complete intersection of a quadric and a cubic, where $n$ is even. Then $Y$ has a multiplicative Chow-Künneth decomposition.

Proof. Let us first construct a CK decomposition for $Y$. Letting $h \in A^{1}(Y)$ denote a hyperplane section, as before we consider

$$
\begin{aligned}
\pi_{Y}^{2 j} & :=\frac{1}{6} h^{n-2-j} \times h^{j} \quad\left(j \neq \frac{n-2}{2}\right), \\
\pi_{Y}^{n-2} & :=\Delta_{Y}-\sum_{j \neq \frac{n-2}{2}} \pi_{Y}^{2 j} \quad \in A^{n-2}(Y \times Y) .
\end{aligned}
$$


We observe that this CK decomposition is generically defined with respect to the family $\mathcal{Y} \rightarrow B$ (Notation 3.3), i.e. it is obtained by restriction from "universal projectors" $\pi_{\mathcal{Y}}^{j} \in A^{n-2}\left(\mathcal{Y} \times{ }_{B} \mathcal{Y}\right)$. (This is just because $h$ and $\Delta_{Y}$ are generically defined.)

Writing $h^{j}(Y):=\left(Y, \pi_{Y}^{j}\right) \in \mathcal{M}_{\text {rat }}$, we have

$$
h^{2 j}(Y) \cong \mathbb{1}(-j) \text { in } \mathcal{M}_{\text {rat }} \quad\left(j \neq \frac{n-2}{2}\right),
$$

i.e. the interesting part of the motive of $Y$ is contained in $h^{n-2}(Y)$.

What we need to prove is that this CK decomposition is MCK, i.e.

$$
\pi_{Y}^{k} \circ \Delta_{Y}^{s m} \circ\left(\pi_{Y}^{i} \times \pi_{Y}^{j}\right)=0 \text { in } A^{2 n-4}(Y \times Y \times Y) \text { for all } i+j \neq k,
$$

or equivalently that

$$
h^{i}(Y) \otimes h^{j}(Y) \stackrel{\Delta_{Y}^{s m}}{\longrightarrow} h(Y)
$$

coincides with

$$
h^{i}(Y) \otimes h^{j}(Y) \stackrel{\Delta_{Y}^{s m}}{\longrightarrow} h(Y) \rightarrow h^{i+j}(Y) \rightarrow h(Y)
$$

for all $i, j$.

We observe that the cycles in (3) are generically defined, and that the vanishing (3) holds true modulo homological equivalence. As such, the required vanishing (3) follows from the Franchetta property for $\mathcal{Y}^{3 / B} \rightarrow B$ in codimension $2 n-4$ (Proposition 3.11 ).

\section{CONSEQUences}

\subsection{Chow ring of $Y$.}

Corollary 5.1. Let $Y \subset \mathbb{P}^{n}$ be as in Theorem 4.1 Then

$$
\operatorname{Im}\left(A^{i}(Y) \otimes A^{j}(Y) \rightarrow A^{i+j}(Y)\right)=\mathbb{Q}\left[h^{i+j}\right] \forall i, j>0 .
$$

Proof. (NB: This argument is similar to [6, Theorem 1.0.1], which is about cubic hypersurfaces.)

We begin by noting that when $n=4$, the complete intersection $Y$ is a $\mathrm{K} 3$ surface and so Corollary 5.1 is already known from the seminal work of Beauville-Voisin [3]. We now suppose that $n \geq 6$, and so $Y$ is a Fano variety.

Let us consider the modified small diagonal

$$
\begin{aligned}
\Gamma_{3}:=\Delta_{Y}^{s m}-\frac{1}{6}\left(\left(p_{12}\right)^{*}\left(\Delta_{Y}\right) \cdot\left(p_{3}\right)^{*}(h)+\left(p_{13}\right)^{*}\left(\Delta_{Y}\right) \cdot\left(p_{2}\right)^{*}(h)+\left(p_{23}\right)^{*}\left(\Delta_{Y}\right) \cdot\left(p_{1}\right)^{*}(h)\right) \\
+\sum_{i+j+k=2 n-4} a_{i j k}\left(p_{1}\right)^{*}\left(h^{i}\right) \cdot\left(p_{2}\right)^{*}\left(h^{j}\right) \cdot\left(p_{3}\right)^{*}\left(h^{k}\right) \in A^{2 n-4}(Y \times Y \times Y),
\end{aligned}
$$

where $a_{i j k} \in \mathbb{Q}$. We make the following claim:

Claim 5.2. There exist $a_{i j k} \in \mathbb{Q}$ such that

$$
\Gamma_{3}=0 \text { in } A^{2 n-4}(Y \times Y \times Y)
$$

for all $Y$ as in Theorem 4.1 . 
The claim implies the corollary, as can be seen by letting $\Gamma_{3}$ act on $\alpha \times \beta \in A^{i+j}(Y \times Y)$ (cf. [6, Lemma 2.0.2]; this uses that $Y$ is Fano so that $A_{0}(Y) \cong \mathbb{Q}$ ). To establish the claim, we reason as in [8, Proof of Proposition 2.8]: the MCK decomposition (plus the fact that $Y$ has transcendental cohomology, plus the relation of Lemma 3.8) yields an identity of the form

$$
\begin{array}{r}
\Delta_{Y}^{s m}=\frac{1}{6}\left(\left(p_{12}\right)^{*}\left(\Delta_{Y}\right) \cdot\left(p_{3}\right)^{*}(h)+\left(p_{13}\right)^{*}\left(\Delta_{Y}\right) \cdot\left(p_{2}\right)^{*}(h)+\left(p_{23}\right)^{*}\left(\Delta_{Y}\right) \cdot\left(p_{1}\right)^{*}(h)\right) \\
+P\left(\left(p_{1}\right)^{*}(h),\left(p_{2}\right)^{*}(h),\left(p_{3}\right)^{*}(h)\right) \text { in } A^{2 n-4}\left(Y^{3}\right),
\end{array}
$$

where $P$ is a symmetric polynomial with $\mathbb{Q}$-coefficients.

(Alternatively, one could also establish Claim 5.2 by using the Franchetta property for $Y^{3}$ in codimension $2 n-4$ (Proposition 3.11); thus one is reduced to finding $a_{i j k}$ such that the claim is verified modulo homological equivalence; this can be done as in [6, Lemma 2.0.1].)

Remark 5.3. In case $n=4, Y$ is a $\mathrm{K} 3$ surface and in this case Corollary 5.1 follows from the seminal work of Beauville-Voisin [3].

For $n$ large, Corollary 5.1 seems to be non-trivial, because it is (expected but) not known that $A^{j}(Y) \cong \mathbb{Q}$ for all $j<(n-2) / 2$.

\subsection{The tautological ring.}

Corollary 5.4. Let $Y \subset \mathbb{P}^{n}$ be a smooth dimensionally transverse intersection of a quadric and a cubic, where $n$ is even. Let $m \in \mathbb{N}$. Let

$$
R^{*}\left(Y^{m}\right):=\left\langle\left(p_{i}\right)^{*}(h),\left(p_{i j}\right)^{*}\left(\Delta_{Y}\right)\right\rangle \subset A^{*}\left(Y^{m}\right)
$$

be the $\mathbb{Q}$-subalgebra generated by pullbacks of the polarization $h \in A^{1}(Y)$ and pullbacks of the diagonal $\Delta_{Y} \in A^{n-2}(Y \times Y)$. (Here $p_{i}$ and $p_{i j}$ denote the various projections from $Y^{m}$ to $Y$ resp. to $Y \times Y$ ). The cycle class map induces injections

$$
R^{*}\left(Y^{m}\right) \hookrightarrow H^{*}\left(Y^{m}, \mathbb{Q}\right) \text { for all } m \leq 2 b-1,
$$

where $b:=\operatorname{dim} H^{n-2}(Y, \mathbb{Q})$.

Moreover, $R^{*}\left(Y^{m}\right) \rightarrow H^{*}\left(Y^{m}, \mathbb{Q}\right)$ is injective for all $m$ if and only if $Y$ is Kimura finitedimensional [18].

Proof. This is directly inspired by the analogous result for cubic hypersurfaces [8, Section 2.3], which in turn is inspired by analogous results for hyperelliptic curves [34], [35] (cf. Remark 5.5 below) and for K3 surfaces [38].

As in [8, Section 2.3], let us write $o:=\frac{1}{6} h^{n-2} \in A^{n-2}(Y)$, and

$$
\tau:=\Delta_{Y}-\frac{1}{6} \sum_{j=0}^{n-2} h^{j} \times h^{n-2-j} \in A^{n-2}(Y \times Y)
$$

(this cycle $\tau$ is nothing but the projector on the motive $h_{\text {prim }}^{n-2}(Y)$ ). Moreover, let us write

$$
\begin{aligned}
o_{i} & :=\left(p_{i}\right)^{*}(o) \in A^{n-2}\left(Y^{m}\right), \\
h_{i} & :=\left(p_{i}\right)^{*}(h) \in A^{1}\left(Y^{m}\right), \\
\tau_{i j} & :=\left(p_{i j}\right)^{*}(\tau) \in A^{n-2}\left(Y^{m}\right) .
\end{aligned}
$$


We now define the $\mathbb{Q}$-subalgebra

$$
\bar{R}^{*}\left(Y^{m}\right):=\left\langle o_{i}, h_{i}, \tau_{i j}\right\rangle \quad \subset H^{*}\left(Y^{m}, \mathbb{Q}\right) \quad(1 \leq i \leq m, 1 \leq i<j \leq m) ;
$$

this is the image of $R^{*}\left(Y^{m}\right)$ in cohomology. One can prove (just as [8, Lemma 2.11] and [38, Lemma 2.3]) that the $\mathbb{Q}$-algebra $\bar{R}^{*}\left(Y^{m}\right)$ is isomorphic to the free graded $\mathbb{Q}$-algebra generated by $o_{i}, h_{i}, \tau_{i j}$, modulo the following relations:

$$
\begin{gathered}
o_{i} \cdot o_{i}=0, \quad h_{i} \cdot o_{i}=0, \quad h_{i}^{n-2}=6 o_{i} ; \\
\tau_{i j} \cdot o_{i}=0, \quad \tau_{i j} \cdot h_{i}=0, \quad \tau_{i j} \cdot \tau_{i j}=(b-1) o_{i} \cdot o_{j} \\
\tau_{i j} \cdot \tau_{i k}=\tau_{j k} \cdot o_{i} ; \\
\sum_{\sigma \in \mathfrak{S}_{b}} \operatorname{sign}(\sigma) \prod_{i=1}^{b} \tau_{i, b+\sigma(i)}=0,
\end{gathered}
$$

To prove Corollary 5.4 it suffices to check that these relations are verified modulo rational equivalence. The relations (4) take place in $R^{*}(Y)$ and so they follow from the Franchetta property for $Y$. The relations (5) take place in $R^{*}\left(Y^{2}\right)$. The first and the last relations are trivially verified, because one may assume $n>4$ and so $Y$ is Fano and one has $A^{2 n-4}\left(Y^{2}\right)=\mathbb{Q}$. As for the second relation of (5), this is the relation of Lemma 3.8,

Relation (6) takes place in $R^{*}\left(Y^{3}\right)$ and follows from the MCK decomposition (Theorem 4.1). Indeed, we have

$$
\Delta_{Y}^{s m} \circ\left(\pi_{Y}^{n-2} \times \pi_{Y}^{n-2}\right)=\pi_{Y}^{2 n-4} \circ \Delta_{Y}^{s m} \circ\left(\pi_{Y}^{n-2} \times \pi_{Y}^{n-2}\right) \text { in } A^{2 n-4}\left(Y^{3}\right),
$$

which (using Lieberman's lemma) translates into

$$
\left(\pi_{Y}^{n-2} \times \pi_{Y}^{n-2} \times \Delta_{Y}\right)_{*} \Delta_{Y}^{s m}=\left(\pi_{Y}^{n-2} \times \pi_{Y}^{n-2} \times \pi_{Y}^{2 n-4}\right)_{*} \Delta_{Y}^{s m} \text { in } A^{2 n-4}\left(Y^{3}\right),
$$

which means that

$$
\tau_{13} \cdot \tau_{23}=\tau_{12} \cdot o_{3} \text { in } A^{2 n-4}\left(Y^{3}\right) .
$$

Finally, relation (7), which takes place in $R^{*}\left(Y^{2 b}\right)$ is the Kimura finite-dimensionality relation [18]: in the notation of loc. cit., relation (7) is written as

$$
\wedge^{b} \pi_{Y}^{n-2, \text { prim }}=0 \text { in } H^{b(2 n-4)}\left(Y^{b} \times Y^{b}, \mathbb{Q}\right),
$$

which expresses the fact that $\left(\operatorname{dim} H_{\text {prim }}^{n-2}(Y, \mathbb{Q})=b-1\right.$ and so $)$

$$
\wedge^{b} H_{\text {prim }}^{n-2}(Y, \mathbb{Q})=0 \text {. }
$$

Assuming that $Y$ is Kimura finite-dimensional, this relation is also verified modulo rational equivalence.

Remark 5.5. Given any curve $C$ and an integer $m \in \mathbb{N}$, one can define the tautological ring

$$
R^{*}\left(C^{m}\right):=\left\langle\left(p_{i}\right)^{*}\left(K_{C}\right),\left(p_{i j}\right)^{*}\left(\Delta_{C}\right)\right\rangle \subset A^{*}\left(C^{m}\right)
$$


(where $p_{i}, p_{i j}$ denote the various projections from $C^{m}$ to $C$ resp. $C \times C$ ). Tavakol has proven [35, Corollary 6.4] that if $C$ is a hyperelliptic curve, the cycle class map induces injections

$$
R^{*}\left(C^{m}\right) \hookrightarrow H^{*}\left(C^{m}, \mathbb{Q}\right) \text { for all } m \in \mathbb{N} .
$$

On the other hand, there are many (non hyperelliptic) curves for which the tautological rings $R^{*}\left(C^{2}\right)$ or $R^{*}\left(C^{3}\right)$ do not inject into cohomology (this is related to the non-vanishing of the Faber-Pandharipande cycle of Remark 3.9, it is also related to the non-vanishing of the Ceresa cycle, cf. [35, Remark 4.2] and [9, Example 2.3 and Remark 2.4]).

Corollary 5.4 shows that the tautological ring of $Y$ behaves similarly to that of hyperelliptic curves, K3 surfaces and cubic hypersurfaces.

\subsection{The MCK decomposition for $Z$.}

Corollary 5.6. Let $\bar{Z} \subset \mathbb{P}^{n+1}(\mathbb{C})$ be a general nodal cubic, where $n$ is even. Then there is a resolution of singularities $Z \rightarrow \bar{Z}$ such that $Z$ has an MCK decomposition.

Proof. For general $\bar{Z}$, the blow-up $Z \rightarrow \bar{Z}$ with center the node is non-singular. To prove the corollary, we interpret $Z$ as the blow-up of $\mathbb{P}^{n}$ with center $Y$ a smooth complete intersection of a cubic and a quadric (Proposition 2.1), and apply Proposition 2.7 (with $M=\mathbb{P}^{n}$ and $N=Y$ ). Let us check all conditions of Proposition 2.7 are satisfied. In view of Theorem 4.1, conditions (1) and (4) are satisfied. Conditions (2) and (3) are about generically defined cycles on $Y$, and so they follow from Proposition 3.6 .

Acknowledgments. Thanks to Yoyo from bellenana.fr.

\section{REFERENCES}

[1] A. Beauville, Sur l'anneau de Chow d'une variété abélienne. Math. Ann. 273 (1986), 647—651,

[2] A. Beauville, On the splitting of the Bloch-Beilinson filtration, in: Algebraic cycles and motives (J. Nagel and C. Peters, editors), London Math. Soc. Lecture Notes 344, Cambridge University Press 2007 ,

[3] A. Beauville and C. Voisin, On the Chow ring of a K3 surface, J. Alg. Geom. 13 (2004), 417—426,

[4] N. Bergeron and Z. Li, Tautological classes on moduli space of hyperkähler manifolds, Duke Math. J., arXiv:1703.04733,

[5] S. Bloch and V. Srinivas, Remarks on correspondences and algebraic cycles, American Journal of Mathematics Vol. 105, No 5 (1983), 1235-1253,

[6] H. Diaz, The Chow ring of a cubic hypersurface, International Math. Res. Notices,

[7] L. Fu, R. Laterveer and Ch. Vial, The generalized Franchetta conjecture for some hyper-Kähler varieties (with an appendix joint with M. Shen), Journal Math. Pures et Appliquées (9) 130 (2019), 1—35,

[8] L. Fu, R. Laterveer and Ch. Vial, The generalized Franchetta conjecture for some hyper-Kähler varieties, II, arXiv:2002.05490,

[9] L. Fu, R. Laterveer and Ch. Vial, Multiplicative Chow-Künneth decompositions and varieties of cohomological K3 type, Annali Mat. Pura ed Applicata, doi: 10.1007/s10231-020-01052-8,

[10] L. Fu, Z. Tian and Ch. Vial, Motivic hyperkähler resolution conjecture for generalized Kummer varieties, arXiv:1608.04968, 
[11] L. Fu and Ch. Vial, Distinguished cycles on varieties with motive of abelian type and the section property, J. Alg. Geom. 29 (2020), 53-107,

[12] W. Fulton, Intersection theory, Springer-Verlag Ergebnisse der Mathematik, Berlin Heidelberg New York Tokyo 1984,

[13] M. Green and P. Griffiths, An interesting 0-cycle, Duke Math. J. 119 no. 2 (2003), 261-313,

[14] D. Huybrechts, The geometry of cubic hypersurfaces, notes available from www.math.unibonn.de/people/huybrech/Notes.pdf,

[15] A. Iliev, D. Favero and L. Katzarkov, On the Griffiths groups of Fano manifolds of Calabi-Yau Hodge type, Pure and Applied Math. Quarterly 10 (2014), 1-55,

[16] A. Iliev and L. Manivel, Fano manifolds of Calabi-Yau type, Journal Pure Appl. Algebra 219 (2015), $2225-2244$,

[17] U. Jannsen, On finite-dimensional motives and Murre's conjecture, in: Algebraic cycles and motives (J. Nagel and C. Peters, editors), Cambridge University Press, Cambridge 2007,

[18] S.-I. Kimura, Chow groups are finite dimensional, in some sense, Math. Ann. 331 no 1 (2005), 173-201,

[19] R. Laterveer, A remark on the Chow ring of Küchle fourfolds of type d3, Bulletin Australian Math. Soc. 100 no. 3 (2019), 410-418,

[20] R. Laterveer, Algebraic cycles and Verra fourfolds, Tohoku Math. J. 72 no. 3 (2020), 451-485,

[21] R. Laterveer, Algebraic cycles and Gushel-Mukai fivefolds, Journal Pure Appl. Algebra 225 no. 5 (2021),

[22] R. Laterveer, On the Chow ring of certain Fano fourfolds, Ann. Univ. Paedagog. Crac. Stud. Math. 19 (2020), 39-52,

[23] R. Laterveer, On the Chow ring of Fano varieties of type $S 2$, Abh. Math. Semin. Univ. Hambg. 90 (2020), $17-28$,

[24] R. Laterveer, Algebraic cycles and Fano threefolds of genus 8, preprint,

[25] R. Laterveer and Ch. Vial, On the Chow ring of Cynk-Hulek Calabi-Yau varieties and Schreieder varieties, Canadian Journal of Math. 72 no 2 (2020), 505-536,

[26] C. Lehn, Twisted cubics on singular cubic fourfolds—On Starr's fibration, Math. Zeitschrift 290 (2018), $379-388$,

[27] J. Murre, On a conjectural filtration on the Chow groups of an algebraic variety, parts I and II, Indag. Math. 4 (1993), 177-201,

[28] J. Murre, J. Nagel and C. Peters, Lectures on the theory of pure motives, Amer. Math. Soc. University Lecture Series 61, Providence 2013,

[29] A. Negut, G. Oberdieck and Q. Yin, Motivic decompositions for the Hilbert scheme of points of a K3 surface, arXiv: $1912.09320 \mathrm{v} 1$,

[30] N. Pavic, J. Shen and Q. Yin, On O'Grady's generalized Franchetta conjecture, Int. Math. Res. Notices (2016), 1-13,

[31] T. Scholl, Classical motives, in: Motives (U. Jannsen et alii, eds.), Proceedings of Symposia in Pure Mathematics Vol. 55 (1994), Part 1,

[32] M. Shen and Ch. Vial, The Fourier transform for certain hyperKähler fourfolds, Memoirs of the AMS 240 (2016), no.1139,

[33] M. Shen and Ch. Vial, The motive of the Hilbert cube $X^{[3]}$, Forum Math. Sigma 4 (2016), 55 pp.,

[34] M. Tavakol, The tautological ring of the moduli space $M_{2}^{r t}$, International Math. Research Notices 2014 no. 24 (2014), 6661-6683,

[35] M. Tavakol, Tautological classes on the moduli space of hyperelliptic curves with rational tails, J. Pure Applied Algebra 222 no. 8 (2018), 2040-2062,

[36] Ch. Vial, On the motive of some hyperkähler varieties, J. Reine Angew. Math. 725 (2017), 235-247,

[37] Q. Yin, The generic nontriviality of the Faber-Pandharipande cycle, International Math. Res. Notices 2015 no 5 (2015), 1263-1277,

[38] Q. Yin, Finite-dimensionality and cycles on powers of K3 surfaces, Comment. Math. Helv. 90 (2015), 503-511. 
Institut de Recherche Mathématique Avancée, CNRS - Université de Strasbourg, 7 Rue RENÉ DESCARTES, 67084 STRASBOURG CEDEX, FRANCE.

Email address: robert. laterveer@math. unistra. fr 